\title{
NO TOPOLOGIES CHARACTERIZE DIFFERENTIABILITY AS CONTINUITY
}

\author{
ROBERT GEROCH, ${ }^{1}$ ERWIN KRONHEIMER AND GEORGE MCCARTY ${ }^{2}$
}

\begin{abstract}
Do there exist topologies $\mathcal{U}$ and $\mathcal{U}$ for the set $R$ of real numbers such that a function $f$ from $R$ to $R$ is smooth in some specified sense (e.g., differentiable, $C^{n}$, or $C^{\infty}$ ) with respect to the usual structure of the real line if and only if $f$ is continuous from $\mathcal{U}$ to $\mathcal{U}$ ? We show that the answer is no.
\end{abstract}

First assume $\mathcal{u}=\boldsymbol{V}$ : we establish that if, with respect to a single given topology on $R$, (a) all functions of the form $x \sim-p x+q(p, q \in R)$ are continuous, and (b) some nonzero function $h$ from $R$ to $R$ which vanishes on the negative numbers is continuous, then the function $k: x \min |x|$ is also continuous.

To prove this we may evidently assume the given topology is not indiscrete. Consequently we can, using (a), find an open set $U$ to include any chosen nonzero $w$ and to exclude 0 . We choose $w$ in $h[R]$ : then $h^{-1}[U]$ is a nonvoid open set containing only nonnegative numbers. Whenever $x \neq 0$, therefore, $|x|$ has, by (a), a neighborhood consisting of nonnegative numbers. For any such neighborhood $N$, however, $k^{-1}[N]=N \cup(-N)$, which, by (a), is a neighborhood of $x$. It follows that $k$ is continuous at $x$. To prove that $k$ is continuous at 0 , note that if $M$ is a neighborhood of 0 so is $M^{*}=M \cap(-M)$, and that $k^{-1}\left[M^{*}\right]=M^{*}$.

The following lemma now shows that there was no loss of generality in the assumption that $\mathfrak{u}=\boldsymbol{v}$.

Lemma. Let $X$ be a set, and let $C(\mathcal{U}, \mathcal{v})$-defined whenever $u, \mathcal{v}$ are topologies on $X$-denote the class of $(\mathcal{U}, \mathcal{V})$-continuous maps. Suppose $C(\mathcal{U}, \mathcal{V})$ is a class $F$ which (i) is closed under composition and (ii) includes the identity map. Then $F=C(\Im, J)$ for some $J$.

Proof. Let $S$ be the topology generated on $X$ by the collection

$$
\left\{f^{-1}[V]: f \in F, V \in v\right\} \text {. }
$$

Then $F \subset C(s, \mathcal{V}) \subset C(\mathcal{U}, \mathcal{V})$; hence $F=C(s, \mathcal{V})$. Let $J$ be the topology

Received by the editors August 8, 1969.

AMS 1969 subject classifications. Primary 5460; Secondary 2641.

Key words and phrases. Differentiability.

${ }^{1}$ Present address: Department of Physics, University of Texas, Austin. NSF postdoctoral fellow.

2 Research supported by NSF Grant GP-7913. 
274 ROBERT GEROCH, ERWIN KRONHEIMER AND GEORGE MCCARTY

$$
\left\{T: f \in F \Rightarrow f^{-1}[T] \in s\right\} .
$$

Then $F \subset C(\mathcal{S}, \mathfrak{J}) \subset C(\mathcal{S}, \mho)$; hence $F=C(\mathcal{S}, \mathfrak{J})$. By (i), each generator of $\delta$ belongs to $J$ : consequently $\delta \subset J$. By (ii), therefore, $\delta=J$.

The following question remains open: Does there exist a topology on $R$ with respect to which a function is continuous if and only if it is analytic?

Birkbeck College, London, England 